\title{
AVALIAÇÃO DE JUSTIÇA E INTENÇÃO DE TURNOVER EM EQUIPES DE VENDAS: TESTE DE UM MODELO TEÓRICO
}

\author{
EVALUATION OF JUSTICE AND TURNOVER INTENTION IN SALES TEAMS: TEST OF A \\ THEORETICAL MODEL
}

EVALUATION OF JUSTICE AND TURNOVER INTENTION IN SALES TEAMS: TEST OF A THEORETICAL MODEL

\section{RESUMO}

O tema da justiça no contexto da gestão de uma equipe de vendas, embora importante para medir o relacionamento e a efetividade dessas equipes, é pouco ou nada explorado no Brasil. O principal propósito deste trabalho é entender a relação entre a avaliação de justiça em sua constituição tetradimensional (informacional, procedimental, distributiva e interpessoal) e a motivação, a lealdade e a intenção de turnover de equipes de vendas. O estudo de campo foi realizado por meio de um survey eletrônico com uma amostra final de 194 respondentes, todos profissionais de vendas. Uma contribuição acadêmica do estudo foi propor uma nova escala para mensuração da justiça percebida. Outra contribuição foi possibilitar o aprofundamento da compreensão da multidimensionalidade da percepção de justiça, lealdade, motivação e intenção de turnover. Nesse sentido, evidenciou-se a influência da justiça percebida na ligação psicológica da equipe de vendas com a empresa.

PALAVRAS-CHAVE Avaliação de justiça, justiça percebida, motivação, lealdade, intenção de turnover.

\section{Evandro Luiz Lopes elldijo@uol.com.br}

Professor do Programa de Pós-Graduação em Administração da Universidade Nove de Julho - São Paulo - SP, Brasil

Sérgio Luiz do Amaral Moretti sergiomoretti@uol.com.br

Professor do Programa de Pós-Graduação em Administração da Universidade Nove de Julho - São Paulo - SP, Brasil

Thomas Brashear Alejandro brashear@mktg.umass.edu

Professor na Isenberg School of Management, University of Massachusetts Amherst - Amherst - MA, United States of America

\begin{abstract}
The theme of justice within in the context of the management of a sales team, albeit important for measuring the relationship and effectiveness of these teams, is little or never explored in Brazil. The main purpose of this work is to understand the relationship between the evaluation of justice in its four-dimensional constitution (informational, procedural, distributive and inter-personal) and the motivation, loyalty and turnover intention of sales teams. The field study was carried out by means of an electronic survey with a final sample of 194 respondents, all sales professionals. One of the academic contributions of the study was to propose a new scale for measuring perceived justice. Another contribution was that of enabling a more in-depth understanding of the multi-dimensional aspects of the perception of justice, loyalty, motivation and turnover intention. In this sense it provided evidence of the perceived justice in the psychological link between the sales team and the company.

keywords Evaluation of justice, perceived justice, motivation, loyalty, turnover intention.

Resumen El tema de la justicia en el contexto de la gestión de un equipo de ventas, aunque importante para medir el relacionamiento y la efectividad de estos equipos, poco o nada se explora en el Brasil. El principal propósito de este trabajo es comprender la relación entre la evaluación de justicia en su formación de cuatro dimensiones (informacional, procedimental, distributiva y interpersonal) y la motivación, la lealtad y la intención de turnover de equipos de ventas. El estudio de campo fue hecho por medio de un survey electrónico con una muestra final de 194 encuestados, todos profesionales de ventas. Una contribución académica del estudio fue proponer una nueva escala para mensuración de la justicia percibida. Otra contribución fue hacer posible una mejor comprensión de la multidimensionalidad de la percepción de justicia, lealtad, motivación e intención de turnover. En ese sentido, evidencióse la influencia de la justicia percibida en la conexión psicológica del equipo de ventas con la empresa.
\end{abstract}

Palabras clave Evaluación de justicia, justicia percibida, motivación, lealtad, intención de turnover. 


\section{INTRODUÇÃO}

A importância desse estudo insere-se no campo do equilíbrio das atividades de vendas e na manutenção de equipes experientes, evitando-se o custo excessivo do turnover. O desenvolvimento de boas relações (KASHYAP e outros, 2007) entre vendedores e seus gestores é fundamental para o sucesso das organizações de vendas (MAERTZ JUNIOR e outros, 2007). A percepção de justiça varia segundo expectativas que são comparadas com os resultados obtidos e construídas durante esse processo (MARTINEZ-TUR e outros, 2006). A volatilidade dessas variáveis não permite o estabelecimento de consensos prima facie, que são impregnados de forte característica relacional e multidimensional (BRASHEAR e outros, 2003; KASHYAP e outros, 2007).

Os vendedores encontram-se em situações em que seus gerentes de vendas têm autoridade para aplicar recompensas ou punições por meio de alterações nos objetivos de vendas e responsabilidade por áreas potenciais (WEITZ, CASTLEBERRY, TANNER, 2004) ou avaliações de desempenho (DECARLO e LEIGH, 1996; PODSAKOFF e MACKENZIE, 1994). Tal poder cria relações assimétricas entre vendedores e gerentes, pelas quais há potencial para a exploração do parceiro mais fraco (KUMAR, SCHEER, STEENKAMP, 1995).

Quando os parceiros mais fracos são incapazes de assegurar as garantias na forma de contratos ou de outras defesas jurídicas, muitas vezes têm de confiar no senso de justiça desses parceiros fortes (ANDERSON e WEITZ, 1992). Injustiças nas interações entre vendedores e gerente de vendas podem influenciar negativamente a satisfação, comprometimento e desempenho na equipe de vendas (PODSAKOFF e MACKENZIE, 1994). Por essa razão, a justiça no trabalho é uma preocupação fundamental para os vendedores.

Justiça no trabalho é uma questão-chave na gestão das organizações (FOLGER e KONOVSKY, 1989; TYLER, 1994). Ao contrário da brasileira, a produção acadêmica norte-americana é extensiva na avaliação da relação entre a percepção dos mecanismos de justiça e de trabalho relacionados com os resultados (MARTIN e HARDER, 1994; TYLER, 1994). Contudo, na área de gestão de vendas, a pesquisa sobre os efeitos da justiça é limitada, embora Podsakoff e MacKenzie (1994) tenham notado que a percepção de justiça é importante para manter uma força de vendas satisfeita e produtiva.

O principal propósito deste estudo foi entender a relação entre a avaliação de justiça, em sua constituição tetradimensional (informacional, processual, distributiva e interpessoal), e três variáveis consequentes: a motivação, a lealdade e a intenção de turnover dos profissionais de vendas envolvidos. As variáveis dependentes escolhidas (motivação, lealdade e intenção de turnover) são importantes na literatura de organização e interação social e, também, na literatura de gestão de vendas (LUO, 2006), todavia são pouco estudadas, visto que os parcos trabalhos publicados investigaram a influência da justiça, em uma constituição bidimensional, na satisfação com o trabalho (BRASHEAR, MANOLIS, BROOKS, 2005; COLQUITT e outros, 2001; KONOVSKY, 2000).

Em alguns dos estudos, verificou-se que a influência da justiça distributiva na satisfação com o trabalho é mais positiva do que a influência da justiça processual com a satisfação (KONOVSKY, 2000; COLQUITT e outros, 2001); no entanto, esse resultado não tem sido encontrado em todos os casos (BRASHEAR, MANOLIS, BROOKS, 2005). Na literatura de vendas, Netemeyer e outros (1997) encontraram uma relação positiva entre capital e satisfação.

O trabalho trouxe contribuições acadêmicas, gerenciais e teóricas. No primeiro caso, pode-se validar uma escala sobre justiça em vendas no Brasil; no segundo, algumas confirmações do estudo podem auxiliar a gestão de vendas a reduzir o turnover de suas equipes; e, finalmente, algumas proposições teóricas puderam ser comprovadas, como foi o caso da justiça distributiva (DEUTSCH, 1975; TYLER, 1994) e interpessoal (BLODGETT, HILL, TAX, 1997; GOODWIN e ROSS, 1992). Por outro lado, não se confirmaram os resultados quanto à justiça informacional (CROPANZANO e GREENBERG, 1997; GREENBERG, 1993) e processual (TAX, BROWN, CHANDRASSHEKARAN, 1998).

O artigo começa com uma breve revisão de estudos anteriores de avaliações de justiça, seguida pelo desenvolvimento do modelo teórico. São desenvolvidas hipóteses referentes às avaliações de justiça pela equipe de vendas. A terceira seção fornece uma breve descrição dos procedimentos metodológicos utilizados e da composição da amostra. Os resultados são descritos na seção quarta, seguidos por uma discussão dos resultados e considerações finais.

\section{CONCEITUAÇÃO DE JUSTIÇA}

O conceito de justiça está diretamente relacionado com o paradigma da desconfirmação de expectativas, que supõe uma transformação consciente da informação 
na qual os indivíduos comparam as suas expectativas com os resultados obtidos - quer sejam de produtos, serviços ou transações - gerando sentenças de satisfação e insatisfação (MARTINEZ-TUR e outros, 2006; OLIVER, 1993). Santos e Fernandes (2008) afirmam que a teoria de justiça foi criada com base nos estudos sobre a transação social e que contribui de maneira decisiva na resolução de conflitos. Esse aporte teórico está embasado em relevantes estudos sobre o aspecto da justiça no tratamento de recuperação de falhas.

O estudo de Blodgett, Wakefield e Barnes (1995) fornece um amplo campo de análise sobre o conceito da justiça, afirmando que o construto possui as suas bases na psicologia social, e pode ser amplamente empregado com sucesso para explicar a reação dos indivíduos a uma variedade de situações de conflito nos mais diferentes contextos (relações trabalhistas, contratuais, promocionais e em operações entre compradores e vendedores). Os autores complementam essa afirmação salientando que a justiça pode ser afetada tanto por aspectos psicológicos (por exemplo, a satisfação, a confiança e a lealdade) como por ações comportamentais (por exemplo, a rotatividade de funcionários, recursos jurídicos, as intenções de recompra).

Em marketing, a justiça tem sido estudada de maneira comedida, embora haja exemplos no estudo da força de vendas com foco em comportamentos de cidadania organizacional (NETEMEYER e outros, 1997), qualidade do relacionamento nos canais de distribuição (KUMAR, SCHEER, STEENKAMP, 1995) e nas respostas organizacionais às queixas do consumidor (BLODGETT, HILL, TAX, 1997). Mesmo que ainda não haja um consenso a respeito da configuração dimensional da justiça, considerou-se, neste trabalho, a modelagem proposta por Blodgett, Hill e Tax (1997) e Tax, Brown e Chandrasshekaran (1998), que a entendem como sendo formada por quatro construtos de segunda ordem: justiça distributiva, justiça processual, justiça informacional e justiça interpessoal, as quais serão detalhadas na sequência dessa revisão.

Já no campo dos estudos organizacionais, as pesquisas sobre o tema da justiça procuraram concentrar-se tanto na importância da criação de uma cidadania organizacional (CHANG e DUBISNKI, 2005; PIERCY e outros, 2006) como nos antecedentes da intenção de turnover com base em estilos de supervisão (MAERTZ JUNIOR e outros, 2007) e nas relações interpessoais, principalmente o que Brashear e outros (2003) e Kashyap e outros (2007) denominam relationalism, ou o elo forte do processo é a relação entre os diversos elementos. Pode-se destacar, também, estudos que procuraram relacionar eficiência e esforço da equipe de vendas com o apoio dos líderes (JARAMILLO e MULKI, 2008) e da percepção de um pagamento justo (RAMASWAMI e SINGH, 2003). O modelo defendido neste estudo concentrou-se nos construtos de justiça distributiva, processual, informacional e interpessoal, como se verá a seguir.

\section{Justiça distributiva}

A justiça distributiva tem origem nos estudos de troca social (BLAU, 1964) e da teoria do capital social (ADAMS, 1965), pela qual os indivíduos maximizam seus recursos por meio de interações (TYLER, 1994). Indivíduos avaliam investimentos e resultados das interações com indivíduos ou organizações, esperando igualar ou superar os investimentos, nesse caso, tempo, dinheiro ou esforço investido em um relacionamento (KUMAR, SCHEER, STEENKAMP, 1995; THIBAUT e WALKER, 1975). Indivíduos que percebem as situações proporcionando maiores retornos para investimentos perceberão, também, que a distribuição das recompensas é suficiente ou justa (GREENBERG, 1988).

Percepções de justiça distributiva podem ter três componentes de desfecho: equidade, igualdade ou necessidades (DEUTSCH, 1975). A necessidade refere-se à percepção do que foi recebido pelo destinatário como algo realmente necessário. A igualdade descreve situações em que todos os indivíduos recebem o mesmo tratamento. Equidade, o componente mais estudado (TYLER, 1994), refere-se à percepção da relação entre insumos e resultados, são as recompensas proporcionais ou justas.

A justiça distributiva enfatiza que o princípio da necessidade de obtenção do resultado está em saber se ele satisfaz os requisitos do destinatário, enquanto a igualdade requer entender se todas as partes (reclamante e reclamado) receberam o mesmo resultado, independentemente das contribuições do ato da reclamação (DEUTSCH, 1975). O autor ressalta ainda que, por conta de sua ancoragem na teoria de intercâmbio, os estudos de marketing têm-se centrado, normalmente, sobre o capital próprio e a regra de distribuição adequada. Vale ressaltar que questões da justiça distributiva são difundidas por toda a sociedade, estando presentes em todas as situações de relacionamento entre indivíduos ou grupos.

Neste estudo, a justiça distributiva é definida como a equidade ou justiça das recompensas recebidas, em 
comparação com os insumos (papéis, responsabilidades e esforços) e em comparação com os benefícios organizacionais gerados com base nesses insumos. A concepção teórica da equidade, bem como seu aspecto distributivo, já foi discutida na literatura de marketing tanto sob a ótica da percepção de custos e benefícios (TAX, BROWN, CHANDRASSHEKARAN, 1998) quanto na intenção de recompra em situações de recuperação de falhas (BLODGETT, HILL, TAX, 1997), além de pesquisas empíricas em ambientes empresariais que possuem altas barreiras de saída, tais como aeroportos e instituições bancárias (SANTOS e FERNANDES, 2008). Entende-se que, sob a ótica de uma equipe de vendas, a dimensão distributiva da justiça se refere à equidade percebida pelos vendedores sobre o resultado tangivel de seu trabalho, ou seja, pelas recompensas oriundas das metas atingidas (BLODGETT, GRANBOIS, WALTERS, 1993). Desse modo, a força de vendas deve perceber a distribuição de recompensas como reconhecimento por bom desempenho, refletindo em motivação e lealdade à organização. Com isso, esperam-se as seguintes relações:

\begin{tabular}{|c|l|}
\hline H1a & $\begin{array}{l}\text { Existe relação positiva entre a avaliação de justiça distri- } \\
\text { butiva e motivação da força de vendas. }\end{array}$ \\
\hline H1b & $\begin{array}{l}\text { Existe relação positiva entre a avaliação de justiça distri- } \\
\text { butiva e a lealdade declarada da força de vendas. }\end{array}$ \\
\hline H1c & $\begin{array}{l}\text { Existe relação negativa entre a avaliação de justiça distri- } \\
\text { butiva e a intenção de turnover da força de vendas. }\end{array}$ \\
\hline
\end{tabular}

\section{Justiça processual}

Justiça processual tem sido conceituada de várias maneiras, mas os componentes principais dizem respeito à equidade das políticas e procedimentos, e à lealdade no processo ou aplicação de procedimentos (LIND e TYLER, 1988; THIBAUT e WALKER, 1975). Algumas pesquisas têm focado apenas as políticas (FOLGER e GREENBERG, 1985) ou sua aplicação (THIBAUT e WALKER, 1975). Outras, ainda, incluíram tanto os procedimentos de avaliação como componentes de interação (AQUINO e outros, 1997), ou combinaram o componente de avaliação com outros construtos: comunicação, refutabilidade, explicação, cognoscibilidade e cortesia (KUMAR, SCHEER, STEENKAMP, 1995; LIND e TYLER, 1988).

Enquanto a justiça distributiva parece ser um conceito bastante complexo, baseado na equidade e proporcionalidade dos resultados, a justiça processu- al baseia-se na operação dos processos (BLODGETT, HILL, TAX, 1997). Apesar de ter como premissa a operacionalização da ocorrência, todos os processos relativos a clientes estão diretamente relacionados com satisfação e qualidade dos serviços (CRONIN e TAYLOR, 1992). Deixando explícito esse relacionamento, Tax, Brown e Chandrasshekaran (1998) relatam que a justiça processual é significativa e deve destinar-se a resolver conflitos de modo a incentivar a continuação do relacionamento entre litigantes, mesmo que os resultados não sejam satisfatórios para nenhuma das partes.

Tyler (1994) centrou-se sobre os procedimentos e sua aplicação incluindo uma variedade de antecedentes da justiça processual (ver também GILLILAND, 1993). Nesse estudo, seguiram-se o trabalho de Tyler (1994) e as sugestões de Thibaut e Walker (1975), que se concentraram na construção global da mensuração da percepção de equidade na gestão das políticas organizacionais. Justiça processual, no contexto de vendas, é definida como a percepção, pelo vendedor, da justiça do gestor no desenvolvimento e aplicação uniforme das políticas e procedimentos. Por isso, espera-se que:

\section{$\mathrm{H} 2 \mathrm{a}$ \\ Existirá relação positiva entre a avaliação de justiça processual e motivação da força de vendas. \\ $\mathrm{H} 2 \mathrm{~b}$ \\ Existirá relação positiva entre a avaliação de justiça pro- cessual e a lealdade declarada da força de vendas. \\ $\mathrm{H} 2 \mathrm{C}$ Existirá relação negativa entre a avaliação de justiça processual e a intenção de turnover da força de vendas.}

\section{Justiça interpessoal}

A justiça interpessoal refere-se à maneira pela qual indivíduos são tratados durante os processos relacionais, isto é, se, durante o relacionamento, os indivíduos são tratados com cortesia e respeito (BIES e SHAPIRO, 1987). A importância do entendimento desse aspecto é evidenciada por Tax, Brown e Chandrashekaran (1998), que ressaltam a importância de explicar o sentimento de injustiça durante o processo, mesmo percebendo a justiça no resultado final. Apesar de os estudos sobre a justiça interpessoal ainda serem embrionários, já que as pesquisas se debruçaram mais sobre as relações de consumo (SOTOMAYOR, 2007), existem evidências de que o tratamento pessoal influencia a percepção de justiça na transação, assim como nos comportamentos posteriores.

Pesquisas anteriores já identificaram como ocorrem as relações entre a justiça interpessoal e várias 
práxis organizacionais - posicionamento de serviços (GOODWIN e ROSS, 1992), avaliações de desempenho funcional, recrutamento e seleção de pessoal (MOHR e BITNER, 1991) - além de terem verificado os relacionamentos entre cortesia, simpatia, sensibilidade, interesse e honestidade entre empregados e empregadores (BLODGETT, HILL, TAX, 1997; GOODWIN e ROSS, 1992) e essa faceta da justiça. Em um segundo momento, verificou-se também a existência de relações positivas entre a justiça interpessoal e os esforços despendidos pelos empregados na resolução de problemas entre as organizações e os consumidores.

Com o entendimento de que a justiça interpessoal reflete a qualidade da interação entre equipe de vendas e supervisores, e de que exerce grande influência no desempenho e na atitude das equipes de trabalho (CASTILHO, 1998), espera-se encontrar que:

\begin{tabular}{c|l} 
H3a & $\begin{array}{l}\text { Existe relação positiva entre a avaliação de justiça inter- } \\
\text { pessoal e motivação da força de vendas. }\end{array}$ \\
$\mathrm{H} 3 b$ & $\begin{array}{l}\text { Existe relação positiva entre a avaliação de justiça inter- } \\
\text { pessoal e a lealdade declarada da força de vendas. }\end{array}$ \\
\hline H3c & $\begin{array}{l}\text { Existe relação negativa entre a avaliação de justiça inter- } \\
\text { pessoal e a intenção de turnover da força de vendas. }\end{array}$
\end{tabular}

\section{Justiça informacional}

Entendida anteriormente como sendo apenas uma faceta da justiça interpessoal (BIES e MOAG, 1986), a percepção de justiça informacional deriva do fornecimento de informações e justificativas das decisões tomadas pelos administradores das organizações (COLQUITT e outros, 2001; KERNAN e HANGES, 2002). Sua relevância é clara quando se avalia que os indivíduos esperam que seus supervisores e gestores lhes prestem explicações claras, lógicas e verdadeiras para as decisões que são tomadas, muito em especial quando os resultados envolvem riscos ou resultados desfavoráveis (CROPANZANO e GREENBERG, 1997; GREENBERG, 1993, 2004).

Sotomayor (2007) reforça esse conceito alegando que as percepções de justiça informacional referem-se à adequação das explicações dadas pelo superior hierárquico, não só no que diz respeito à classificação de desempenho dada ao subordinado, mas também pelas explicações e pelo feedback contínuo fornecido durante o período avaliado. A autora reforça o entendimento da avaliação de desempenho como um dos elementos do ciclo de desempenho, ou seja, na acepção mais estrita de gestão de desempenho.
Estudos anteriores indicam que as informações prévias reduzem as reações negativas dos indivíduos em relação à percepção de injustiça ou riscos (CROPANZANO e GREENBERG, 1997; GREENBERG, 1993), além de contribuírem para o desenvolvimento de atitudes positivas das equipes em relação aos supervisores e suas organizações (CASTILHO, 1998). Com isso, formula-se a última hipótese do estudo:

\begin{tabular}{|l|l|} 
H4a & $\begin{array}{l}\text { Existe relação positiva entre a avaliação de justiça infor- } \\
\text { macional e motivação da força de vendas. }\end{array}$ \\
\hline H4b & $\begin{array}{l}\text { Existe relação positiva entre a avaliação de justiça infor- } \\
\text { macional e a lealdade declarada da força de vendas. }\end{array}$ \\
\hline H4c & $\begin{array}{l}\text { Existe relação negativa entre a avaliação de justiça infor- } \\
\text { macional e a intenção de turnover da força de vendas. }\end{array}$
\end{tabular}

Com base na revisão da literatura realizada, o modelo teórico da pesquisa é apresentado na Figura 1.

O modelo propõe a existência de relações positivas entre as quatro dimensões de justiça percebida com a motivação e com a lealdade e relação negativa entre intenção de turnover e os mesmos construtos consequentes. Essa concepção é inovadora, pois, em estudos anteriores, a justiça é entendida como um construto bidimensional ou, raramente, tridimensional, e, até onde se sabe, seu relacionamento com a lealdade, motivação e intenção de turnover não havia sido testado.

\section{Figura 1 - Modelo teórico do estudo}

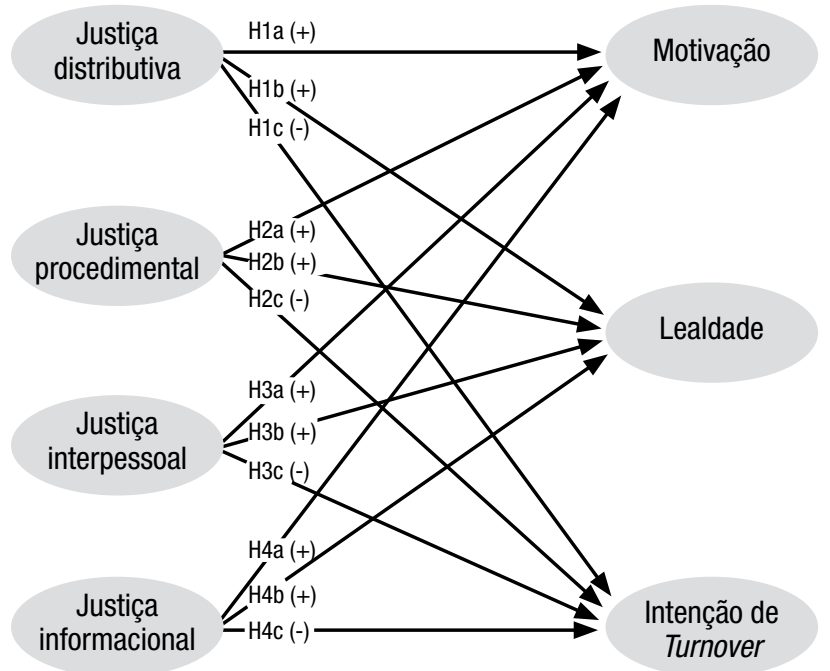




\section{MÉTODO UTILIZADO NA PESQUISA}

Nesta seção, serão descritos os aspectos metodológicos que nortearam a etapa empírica deste trabalho.

\section{Escala}

Um questionário contendo 37 itens foi desenvolvido pelos autores. As quatro variáveis antecedentes foram mensuradas por meio de 21 itens (cinco itens para justiça procedimental, cinco itens para justiça informacional, cinco itens para justiça interpessoal e seis itens para mensuração da justiça distributiva) adaptados dos trabalhos de Brashear, Brooks e Boles (2004) e Brashear, Manolis e Brooks (2005), que tiveram como objetivo a proposição e validação de um modelo integrativo que relacionava 12 construtos, entre os quais a percepção de justiça, benevolência, satisfação, respeito, oportunismo, controle e grau de supervisão, com a intenção de turnover de equipes de vendas norte-americanas, por meio de 119 indicadores. A escala não foi totalmente validada devido à dificuldade de aplicação pela baixa parcimônia do instrumento.
As três variáveis consequentes foram mensuradas por meio de 16 itens (cinco itens para lealdade, cinco itens para motivação e seis itens para mensurar a intenção de turnover). O questionário foi apresentado em formato estruturado e os itens, avaliados por meio de uma escala do tipo de Likert de sete pontos. O Quadro 1 apresenta as variáveis e itens utilizados no estudo.

\section{Amostra e procedimentos}

O questionário foi submetido eletronicamente, por meio do website de pesquisas Questionpro (www. questionpro.com), a um grupo inicial de 200 profissionais de vendas de uma empresa varejista que comercializa produtos para construção civil, utilidades domésticas e produtos para decoração residencial.

Ao final do questionário, solicitava-se que o respondente indicasse o endereço eletrônico de outros profissionais de vendas (máximo 10) que pudessem responder ao mesmo questionário, garantindo que a indicação seria tratada com o devido sigilo. O questionário eletrônico foi acessado por 350 profissionais de vendas durante o tempo em que esteve disponível no website. Com isso, a amostra final foi recrutada por

\section{Quadro 1 - Variáveis e itens utilizados}

\begin{tabular}{|c|c|c|}
\hline Variável & Legenda & Item \\
\hline Lealdade & $\begin{array}{l}\text { LEAL1 } \\
\text { LEAL2 } \\
\text { LEAL3 } \\
\text { LEAL4 } \\
\text { LEAL5 }\end{array}$ & $\begin{array}{l}\text { Minha lealdade para com esta empresa é uma razão importante para eu continuar a trabalhar aqui. } \\
\text { Por causa da minha lealdade para com esta empresa, eu permaneceria nela mesmo em tempos difíceis. } \\
\text { Quero continuar associado(a) a esta empresa por causa da minha fidelidade a ela. } \\
\text { Pretendo continuar a trabalhar nesta empresa porque me sinto como "um membro da família". } \\
\text { Levando em consideração tudo o que aconteceu ao longo dos anos, acho que eu deveria manter o meu vínculo } \\
\text { com esta empresa. }\end{array}$ \\
\hline $\begin{array}{l}\text { Intenção } \\
\text { Turnover }\end{array}$ & $\begin{array}{l}\text { IT1 } \\
\text { IT2 } \\
\text { IT4 } \\
\text { IT5 } \\
\text { IT6 }\end{array}$ & $\begin{array}{l}\text { Eu penso com frequência em deixar este emprego. } \\
\text { Eu pretendo deixar este emprego. } \\
\text { Eu penso com frequência em outro tipo de atividade profissional (um tipo de atividade diferente do que eu faço agora) } \\
\text { Nos próximos } 12 \text { meses, eu pretendo procurar uma atividade para substituir meu emprego atual (outro empre- } \\
\text { go, estudo em tempo integral etc.) } \\
\text { Já procurei um novo emprego. } \\
\text { Estou sempre procurando um emprego melhor. }\end{array}$ \\
\hline Motivação & $\begin{array}{l}\text { MOTIV1 } \\
\text { M0TIV2 } \\
\text { M0TIV3 } \\
\text { M0TIV4 }\end{array}$ & $\begin{array}{l}\text { A maior satisfação da minha vida vem do meu trabalho. } \\
0 \text { que eu faço no meu emprego é muito significativo para mim. } \\
\text { Os resultados do meu trabalho, bons ou ruins, devem ser atribuídos a mim. } \\
\text { Eu gosto de fazer mais do que se espera de mim no meu emprego. }\end{array}$ \\
\hline
\end{tabular}




\begin{tabular}{|c|c|c|}
\hline $\begin{array}{c}\text { Justiça } \\
\text { distributiva }\end{array}$ & $\begin{array}{l}\text { JDIS1 } \\
\text { JDIS2 } \\
\text { JDIS3 } \\
\text { JDIS4 } \\
\text { JDIS5 } \\
\text { JDIS6 }\end{array}$ & $\begin{array}{l}\text { Que tipo de compensação você recebe... } \\
\text {... pelo tempo e pelo esforço que você investiu para apoiar a empresa? } \\
\text {... pelos papéis que tem de desempenhar? } \\
\text {...em comparação com sua contribuição ao esforço de marketing de sua empresa? } \\
\text {...considerando-se as responsabilidades que você tem? } \\
\text {...pelo esforço que você faz? } \\
\text {...pelo trabalho que executa? }\end{array}$ \\
\hline $\begin{array}{c}\text { Justiça } \\
\text { informacional }\end{array}$ & $\begin{array}{l}\text { JINF1 } \\
\text { JINF2 } \\
\text { JINF3 } \\
\text { JINF4 } \\
\text { JINF5 }\end{array}$ & $\begin{array}{l}\text { Meu chefe... } \\
\text { Raramente explica suas decisões. } \\
\text { Apresenta razões válidas para mudanças nas políticas da empresa. } \\
\text { Informa sobre novas diretrizes e suas implicações. } \\
\text { Distribui informações sobre novos processos de tomada de decisões. } \\
\text { Reserva um tempo para explicar mudanças nas políticas da empresa. }\end{array}$ \\
\hline $\begin{array}{c}\text { Justiça } \\
\text { procedimental }\end{array}$ & $\begin{array}{l}\text { JPROC1 } \\
\text { JPROC2 } \\
\text { JPROC3 } \\
\text { JPROC4 } \\
\text { JPROC5 }\end{array}$ & $\begin{array}{l}\text { A empresa encoraja os integrantes da equipe de vendas a cooperarem uns com os outros. } \\
\text { A maior parte da equipe de vendas sabe sobre a produtividade de cada um dos integrantes. } \\
\text { A empresa cria um ambiente em que os integrantes da equipe de vendas respeitam o trabalho de cada um. } \\
\text { A empresa encoraja discussões relacionadas ao trabalho entre o pessoal de vendas. } \\
\text { A maioria das pessoas da equipe de vendas da minha empresa é capaz de avaliar com precisão o trabalho de cada um. }\end{array}$ \\
\hline $\begin{array}{c}\text { Justiça } \\
\text { interpessoal }\end{array}$ & $\begin{array}{l}\text { JINT1 } \\
\text { JINT2 } \\
\text { JINT3 } \\
\text { JINT4 } \\
\text { JINT5 }\end{array}$ & $\begin{array}{l}\text { Quando toma decisões importantes, meu chefe leva em conta o que é bom para mim. } \\
\text { Meu chefe muitas vezes me passou informações que mais tarde se descobriu não serem exatas. } \\
\text { Meu chefe é digno de confiança. } \\
\text { Sempre que meu chefe me dá conselhos sobre minhas atividades de vendas, sei que ele está compartilhando } \\
\text { comigo sua capacidade de analisar. } \\
\text { Se tivesse que definir a forma de tratamento que meu chefe me dispensa, diria que ele(a) é muito justo(a). }\end{array}$ \\
\hline
\end{tabular}

meio da técnica snowball (HAIR JUNIOR e outros, 2005; MALHOTRA, 2006), que consiste na obtenção de novos sujeitos para o estudo por meio da indicação feita pela amostra inicial. A amostra utilizada é classificada como não probabilística por conveniência (MALHOTRA, 2006).

\section{Critérios para análise dos dados}

O banco de dados coletado foi previamente preparado para as análises subsequentes, conforme recomendações de Kline (2005) e Hair Junior e outros (2005), por meio da verificação da normalidade, linearidade, homoscedasticidade e descarte das observações com missing values e também dos outliers. Para a análise dos dados, realizou-se a análise fatorial exploratória (AFE), com a utilização do SPSS 13.0, e análise fato- rial confirmatória (AFC), utilizando-se o Lisrel 8.51 (JÖRESKOG e SÖRBOM, 1993).

A AFE deu-se pelo método de análise de componentes principais e rotação ortogonal varimax. Mesmo sendo razoável pressupor intercorrelações entre as variáveis, optou-se pela rotação ortogonal para a análise mais estrita dos dados. Nessa fase, também foi feita a análise de confiabilidade de consistência interna da escala por meio do cálculo do coeficiente de Alfa de Cronbach. Os seguintes critérios foram adotados para a AFE: carga fatorial superior a 0,5, carga cruzada entre fatores inferior a 0,4, comunalidade extraída dos itens superior a 0,4, teste de esfericidade de Bartlett significativo ao nível de 5\% e teste Kaiser-Meyer-Olkin (KMO) superior a 0,7 (HAIR JUNIOR e outros, 2005). Para o coeficiente de Alfa de Cronbach, o valor mínimo aceitável foi 0,7 (NUNNALLY, 1978). 
A AFC seguiu a metodologia proposta por Anderson e Gerbing (1988), sendo realizada em duas etapas. $\mathrm{Na}$ primeira, foram analisados os modelos de mensuração do conjunto de variáveis, buscando identificar sua validade convergente - o quanto os itens de uma variável latente explicam sua variância total, expressa pelas matrizes Lambda $(\boldsymbol{\lambda})$ superiores a 0,60 - e sua validade discriminante - ou seja, o quanto a variável é independente das demais, expressas pelas matrizes Phi $(\phi)$ inferiores a 0,60 (ANDERSON e GERBING, 1988; KLINE, 2005). Por essa metodologia, os modelos são considerados apropriados quando, além das matrizes Phi e Lambda adequadas, apresentarem os índices de ajustamento CFI, GFI, NFI superiores a 0,90 e o RMR padronizado menor que 0,05 (KLINE, 2005), e, ainda, quando o RMSEA apresentar coeficiente menor que 0,08 para uma adequação razoável ou menor que 0,05 para uma boa adequação (BROWNE e CUDECK, 1993). $\mathrm{Na}$ segunda etapa, foi analisado o modelo estrutural, ou seja, as relações entre as variáveis latentes.

\section{RESULTADOS DA PESQUISA: APRESENTAÇÃO E DISCUSSÃO}

Nesta seção, serão apresentados e discutidos os resultados empíricos observados na coleta dos dados.

\section{Caracterização da amostra}

Como já informado, o questionário foi acessado por 350 visitantes, contudo apenas 304 respondentes (95\% da amostra original) iniciaram seu preenchimento. Todavia, após a preparação do banco de dados descrita na seção anterior, a amostra final deste trabalho foi constituída por 194 respondentes, dos quais 145 são homens $(74,7 \%)$ e 49 (25,3\%) são mulheres, 125 $(64,4 \%)$ são casados(as) e $50(25,8 \%)$, solteiros(as). Os respondentes têm idade média de 36 anos $(\mathrm{dp}=9,8)$, possuem 5,5 anos de trabalho na empresa na qual atuam $(d p=5,0)$ e 12 anos atuando em vendas $(d p=9,2)$, em média. Quanto à renda, 40 respondentes $(20,6 \%)$ alegaram receber até $\mathrm{R} \$ 12.000,00$ anuais, $71(36,6 \%)$ alegaram receber entre $\mathrm{R} \$ 12.000,00$ e $\mathrm{R} \$ 36.000,00$ e $51(26,3)$ informaram ter renda média anual superior a $\mathrm{R} \$ 60.000,00$. A amostra foi basicamente constituída por vendedores de materiais para construção civil (156 respondentes ou 80,4\%) e por vendedores de medicamentos (20 respondentes ou 10,3\%).
Dos 110 questionários desprezados (36\% da amostra de 304 respondentes), 78 (ou 25\%) não foram preenchidos completamente. Acredita-se que, como os convites para o questionário foram enviados para os endereços eletrônicos profissionais, o tempo para resposta ( $\mu=17$ minutos) foi um fator impeditivo. Mesmo diante dessa alta taxa de desistência, a amostra final (194) é considerada adequada para a técnica de análise estatística adotada. Hair Junior e outros (2005) recomendam que, para a utilização da análise fatorial confirmatória, o pesquisador utilize, ao menos, entre três e cinco observações (respondentes) para cada assertiva. Como alternativa, no caso de amostras menores, sugere-se uma amostra mínima contendo 10 vezes o número correspondente ao máximo de coeficientes estruturais associados a uma variável latente. No caso dessa aplicação, as variáveis dependentes possuem quatro indicadores, o que demandaria uma amostra mínima de 40 respondentes. Contudo, com essa situação, deve-se adotar o Partial Least Squares (PLS) como técnica de análise multivariada (GOODHUE, LEWIS, THOMPSON, 2006).

\section{Resultados da análise fatorial exploratória}

Na primeira tentativa, a AFE apontou para a necessidade da eliminação de um item de mensuração da justiça informacional (JINF1 - Meu chefe raramente explica suas decisões) por baixa comunalidade. Após a eliminação desse item, na segunda tentativa, apurou-se KMO de 0,883 e índice de esfericidade de Bartlett significativo a $1 \%\left(\chi^{2}=5176,13 ; 595\right.$ g.l.; $\left.\mathrm{p}<1 \%\right)$. Nessa segunda tentativa, foram identificados sete fatores com eigenvalue superiores a um que confirmaram a configuração teórica dos itens. Como demonstrado no Quadro 2, as cargas fatoriais e a comunalidade extraída de todos os itens foram satisfatórias, assim como o coeficiente de consistência interna da escala. Além disso, a Tabela 1 apresenta a matriz de correlação entre os construtos.

\section{Resultados da análise fatorial confirmatória (AFC)}

O resultado dos modelos de mensuração e estrutural serão expostos a seguir.

\section{Modelos de mensuração}

Os índices de ajustamento do modelo de mensuração das variáveis independentes foram aceitá- 
Quadro 2 - Resultado da análise fatorial exploratória

\begin{tabular}{|c|c|c|c|c|c|c|c|c|}
\hline \multicolumn{9}{|c|}{ Fatores } \\
\hline Item & 1 & 2 & 3 & 4 & 5 & 6 & 7 & Comunalidade \\
\hline LEAL1 & & & & & 0,702 & & & 0,694 \\
\hline LEAL2 & & & & & 0,707 & & & 0,674 \\
\hline LEAL3 & & & & & 0,826 & & & 0,81 \\
\hline LEAL4 & & & & & 0,769 & & & 0,761 \\
\hline LEAL5 & & & & & 0,669 & & & 0,706 \\
\hline IT1 & & 0,787 & & & & & & 0,744 \\
\hline IT2 & & 0,757 & & & & & & 0,720 \\
\hline IT3 & & 0,705 & & & & & & 0,599 \\
\hline IT4 & & 0,833 & & & & & & 0,763 \\
\hline IT5 & & 0,712 & & & & & & 0,686 \\
\hline IT6 & & 0,639 & & & & & & 0,476 \\
\hline M0TIV1 & & & & & & & 0,530 & 0,409 \\
\hline MOTIV2 & & & & & & & 0,764 & 0,692 \\
\hline MOTIV3 & & & & & & & 0,590 & 0,476 \\
\hline MOTIV4 & & & & & & & 0,777 & 0,664 \\
\hline JDIS1 & 0,802 & & & & & & & 0,813 \\
\hline JDIS2 & 0,862 & & & & & & & 0,881 \\
\hline JDIS3 & 0,76 & & & & & & & 0,740 \\
\hline JDIS4 & 0,868 & & & & & & & 0,869 \\
\hline JDIS5 & 0,894 & & & & & & & 0,893 \\
\hline JDIS6 & 0,864 & & & & & & & 0,834 \\
\hline JINF2 & & & & & & 0,667 & & 0,682 \\
\hline JINF3 & & & & & & 0,763 & & 0,783 \\
\hline JINF4 & & & & & & 0,769 & & 0,734 \\
\hline JINF5 & & & & & & 0,738 & & 0,674 \\
\hline JPR0C1 & & & 0,75 & & & & & 0,591 \\
\hline JPROC2 & & & 0,873 & & & & & 0,819 \\
\hline JPR0C3 & & & 0,708 & & & & & 0,597 \\
\hline JPR0C4 & & & 0,832 & & & & & 0,789 \\
\hline JPR0C5 & & & 0,794 & & & & & 0,749 \\
\hline JINT1 & & & & 0,623 & & & & 0,624 \\
\hline JINT2 & & & & 0,798 & & & & 0,824 \\
\hline JINT3 & & & & 0,805 & & & & 0,735 \\
\hline JINT4 & & & & 0,774 & & & & 0,807 \\
\hline JINT5 & & & & 0,729 & & & & 0,790 \\
\hline Eigenvalues & 11,7 & 4,1 & 2,5 & 2,3 & 1,9 & 1,4 & 1,2 & \\
\hline $\begin{array}{l}\text { Variância } \\
\text { explicada }\end{array}$ & $33,50 \%$ & $11,80 \%$ & $7,10 \%$ & $6,60 \%$ & $5,40 \%$ & $3,90 \%$ & $3,40 \%$ & \\
\hline Alfa & 0,95 & 0,87 & 0,89 & 0,91 & 0,88 & 0,86 & 0,75 & \\
\hline
\end{tabular}

veis $\left(\chi^{2}=433,43 / 164\right.$ g.1.; $\mathrm{p}<0,05$, RMSEA $=0,08$; CFI $=0,94 ; \mathrm{GFI}=0,90$; $\mathrm{NFI}=0,90 ; \mathrm{RMR}=0,05)$. As cargas fatoriais padronizadas dos itens (matriz Lambda) ficaram entre 0,64 e 0,93, comprovando a validade convergente, e as cargas fatoriais padronizadas das matrizes Phi ficaram entre 0,44 e 0,57, confirmando a validade discriminante das variáveis. O detalhamento desse modelo de mensuração pode ser verificado na Tabela 2 .

Do mesmo modo, os índices de ajustamento do modelo de mensuração das variáveis dependentes foram aceitáveis $(\chi 2=142,36 / 59$ g.1.; $\mathrm{p}<0,05$, RMSEA=0,08; CFI=0,93; $\mathrm{GFI}=0,90 ; \mathrm{NFI}=0,89 ; \mathrm{RMR}=0,06)$. As cargas fatoriais padronizadas dos itens (matriz Lambda) ficaram entre 0,61 e 0,86, comprovando a validade convergente, e as cargas fatoriais padronizadas das matrizes Phi ficaram entre 0,36 e 0,60, confirmando a validade discriminante das variáveis. O detalhamento desse outro modelo de mensuração pode ser verificado na Tabela 3. Finalizada essa etapa, providenciou-se a modelagem estrutural e a análise dos caminhos correspondente.

\section{Modelo estrutural}

Na primeira tentativa com o modelo estrutural, após a análise dos índices de ajustamento $(\chi 2=943,22 / 533$ g.l.; $\mathrm{p}<0,05$, RMSEA=0,06; CFI=0,91; $\mathrm{GFI}=0,78 ; \mathrm{NFI}=0,82 ; \mathrm{RMR}=0,03) \mathrm{e}$ das cargas fatoriais padronizadas, percebeu-se que oito caminhos não foram significativos. Os resultados indicam que a justiça procedimental e a justiça informacional não se associaram a nenhuma variável dependente. Do mesmo modo, a justiça interpessoal não se associou com a motivação e com a lealdade. Porém, como esperado, a justiça distributiva associou-se positivamente 
Tabela 1 - Matriz de correlação entre os construtos

\begin{tabular}{|c|c|c|c|c|c|c|c|}
\hline & 1 & 2 & 3 & 4 & 5 & 6 & 7 \\
\hline Lealdade & 1,00 & & & & & & \\
\hline Intenção de Turnover & $-0,20$ & 1,00 & & & & & \\
\hline Motivação & 0,13 & $-0,13$ & 1,00 & & & & \\
\hline Justiça procedimental & 0,28 & $-0,16$ & 0,08 & 1,00 & & & \\
\hline Justiça informacional & 0,38 & $-0,28$ & 0,18 & 0,49 & 1,00 & & \\
\hline Justiça interpessoal & 0,37 & $-0,42$ & 0,19 & 0,44 & 0,67 & 1,00 & \\
\hline Justiça distributiva & 0,43 & $-0,39$ & 0,31 & 0,43 & 0,51 & 0,58 & 1,00 \\
\hline
\end{tabular}

Tabela 2 - Validade convergente e discriminante das variáveis independentes

\begin{tabular}{|c|c|c|c|c|}
\hline & Procedimental & Informacional & Interpessoal & Distributiva \\
\hline JPROC1 & 0.64 & & & \\
\hline JPROC2 & 0.85 & & & \\
\hline JPROC3 & 0.68 & & & \\
\hline JPROC4 & 0.91 & & & \\
\hline JPROC5 & 0.86 & & & \\
\hline JINF2 & & 0.77 & & \\
\hline JINF3 & & 0.89 & & \\
\hline JINF4 & & 0.83 & & \\
\hline JINF5 & & 0.67 & & \\
\hline JINT1 & & & 0.73 & \\
\hline JINT2 & & & 0.85 & \\
\hline JINT3 & & & 0.76 & \\
\hline JINT4 & & & 0.89 & \\
\hline JINT5 & & & 0.88 & \\
\hline JDIS1 & & & & 0.88 \\
\hline JDIS2 & & & & 0.93 \\
\hline JDIS3 & & & & 0.81 \\
\hline JDIS4 & & & & 0.90 \\
\hline JDIS5 & & & & 0.93 \\
\hline JDIS6 & & & & 0.88 \\
\hline Justiça procedimental & 1.00 & & & \\
\hline Justiça informacional & 0.50 & 1.00 & & \\
\hline Justiça interpessoal & 0.46 & 0.57 & 1.00 & \\
\hline Justiça distributiva & 0.42 & 0.50 & 0.56 & 1.00 \\
\hline
\end{tabular}




\begin{tabular}{|c|c|c|c|}
\hline \multicolumn{3}{|c|}{$\begin{array}{l}\text { Tabela } 3 \\
\text { discriminante das variáveis dependentes }\end{array}$} \\
\hline & Lealdade & Turnover & Motivação \\
\hline LEAL1 & 0,61 & & \\
\hline LEAL2 & 0,75 & \\
\hline LEAL3 & 0,86 & & \\
\hline LEAL4 & 0,81 & & \\
\hline LEAL5 & 0,80 & & \\
\hline IT2 & & 0,80 & \\
\hline IT3 & & 0,72 & \\
\hline IT4 & & 0,83 & \\
\hline IT5 & & 0,69 & \\
\hline IT6 & & 0,64 & \\
\hline MOTIV1 & & & 0,72 \\
\hline M0TIV2 & & & 0,72 \\
\hline MOTIV3 & & & 0,63 \\
\hline MOTIV4 & & & 0,77 \\
\hline Lealdade & 1,00 & & \\
\hline Turnover & 0,60 & 1,00 & \\
\hline Motivação & 0,50 & 0,36 & 1,00 \\
\hline
\end{tabular}

com a motivação e com a lealdade e negativamente com a intenção de turnover. Além disso, os resultados também indicaram haver relacionamento negativo entre a justiça interpessoal e a intenção de turnover da amostra. As cargas padronizadas estão demonstradas na Tabela 4.

A Figura 2 mostra os caminhos e as cargas fatoriais encontradas.

\section{Discussão dos resultados encontrados}

O primeiro ponto de destaque é a consistência da escala proposta para este estudo. A validade interna dos itens e a adequação fatorial alinhada ao arcabouço teórico considerado credenciam a escala desenvolvida para mensuração das avaliações de justiça procedimental, informacional, interpessoal e distributiva, além da motivação, da lealdade e da intenção de turnover de uma equipe de vendas. A AFE também indicou que a justiça é um construto formado por quatro dimensões distintas, como havia sido previsto.

Em segundo lugar, os resultados indicaram que a justiça distributiva e a justiça interpessoal têm forte correlação com manutenção da estabilidade da equipe de vendas. Já era de se esperar que uma boa avaliação da justiça distributiva, por parte da força de vendas, se traduzisse na intenção de permanecer na empresa, pois o perfil de um vendedor, que geralmente é um profissional motivado por recompensas financeiras, está totalmente alinhado a esse pressuposto. Contudo, a relação entre a justiça interpessoal e intenção de turnover $(\mathrm{t}(194)=2,91 ; \mathrm{p}<1 \%)$ foi ainda maior que a relação entre justiça distributiva e a intenção de turnover $(\mathrm{t}(194)=2,70 ; \mathrm{p}<1 \%)$. Isso salienta a importância da manutenção de um bom ambiente de trabalho, no qual os gestores devem reforçar o aspecto de compromisso com sua equipe de vendas.

Curiosamente, a justiça interpessoal não se associou à lealdade e a motivação, mas, como previsto, associou-se negativamente à intenção de turnover. Esse resultado indica que a força de vendas encara a justiça interpessoal como um fator higiênico, isto é, sua existência é condição essencial para a permanência na organização, porém não gera motivação ou fidelidade.

Um terceiro ponto de destaque é o da comprovação empírica dos relacionamentos entre justiça distributiva e a motivação, a lealdade e a intenção de turnover da equipe de vendas. Uma forte característica do profissional de vendas é a importância que este atribui para a recompensa monetária por seus esforços, por comissões ou premiações a esforços adicionais (ativação de clientes ou negócios extraordinários). Ficou evidente que a percepção de justiça aumenta quando existe remuneração salarial e distribuição de uma parte dos lucros gerados e que estes exercerão forte influência na motivação e na lealdade da equipe de vendas, além de minimizar os riscos de uma possível saída dos profissionais.

O quarto aspecto que cabe salientar é a não relação entre a avaliação da justiça informacional e da justiça procedimental com as variáveis consequentes do modelo. Os procedimentos adotados pelas organizações e a percepção de que as informações fornecidas pelos supervisores são frequentes, corretas e razoáveis não explicou a motivação, a lealdade e a intenção de turnover dos vendedores. Um dos possíveis motivos para esse resultado é o ambiente de competição intensa que rodeia a força de vendas. Por sofrer pressões externas - tais como concorrência e fatores econômicos - e internas - competição com pares, cobrança sobre metas, além de problemas operacionais - os vendedores ancoram sua motivação, lealdade e vontade de permanecer na organização muito mais na remuneração e premiações em decorrência 


\begin{tabular}{|c|c|c|c|c|c|}
\hline Hipótese & Caminho & $\begin{array}{c}\text { Carga } \\
\text { padronizada }\end{array}$ & Teste $\mathbf{t}$ & Valor $p$ & $\begin{array}{l}\text { Teste da } \\
\text { hipótese }\end{array}$ \\
\hline $\mathrm{H} 1 \mathrm{a}$ & Justiça distributiva --> Motivação & 0,31 & 2,69 & $<1 \%$ & não rejeitada \\
\hline $\mathrm{H} 1 \mathrm{~b}$ & Justiça distributiva --> Lealdade & 0,29 & 3,1 & $<1 \%$ & não rejeitada \\
\hline $\mathrm{H} 1 \mathrm{C}$ & Justiça distributiva --> Intenção de turnover & $-0,25$ & 2,7 & $<1 \%$ & não rejeitada \\
\hline $\mathrm{H} 2 \mathrm{a}$ & Justiça procedimental --> Motivação & $-0,08$ & 0,82 & n.s. & rejeitada \\
\hline $\mathrm{H} 2 \mathrm{~b}$ & Justiça procedimental --> Lealdade & 0,04 & 0,53 & n.s. & rejeitada \\
\hline $\mathrm{H} 2 \mathrm{C}$ & Justiça procedimental --> Intenção de turnover & 0,08 & 0,89 & n.s. & rejeitada \\
\hline $\mathrm{H} 3 \mathrm{a}$ & Justiça interpessoal --> Motivação & 0,01 & 0,06 & n.s. & rejeitada \\
\hline $\mathrm{H} 3 \mathrm{~b}$ & Justiça interpessoal--> Lealdade & 0,08 & 0,72 & n.s. & rejeitada \\
\hline $\mathrm{H} 3 \mathrm{c}$ & Justiça interpessoal --> Intenção de turnover & $-0,33$ & 2,91 & $<1 \%$ & não rejeitada \\
\hline $\mathrm{H} 4 \mathrm{a}$ & Justiça informacional --> Motivação & 0,05 & 0,42 & n.s. & rejeitada \\
\hline $\mathrm{H} 4 \mathrm{~b}$ & Justiça informacional --> Lealdade & 0,16 & 1,43 & n.s. & rejeitada \\
\hline $\mathrm{H} 4 \mathrm{C}$ & Justiça informacional --> Intenção de turnover & 0,03 & 0,29 & n.s. & rejeitada \\
\hline
\end{tabular}

Os valores críticos para $t$ são: 1,64 para $p<0,10 ; 1,96$ para $p<0,05 ; 2,58$ para $p<0,01$ e 3,29 para $p<0,001$. Obs. n.s. - não significante ao nível de $10 \%$ de confiança

\section{Figura 2 - Carga fatorial e caminhos do modelo estrutural}

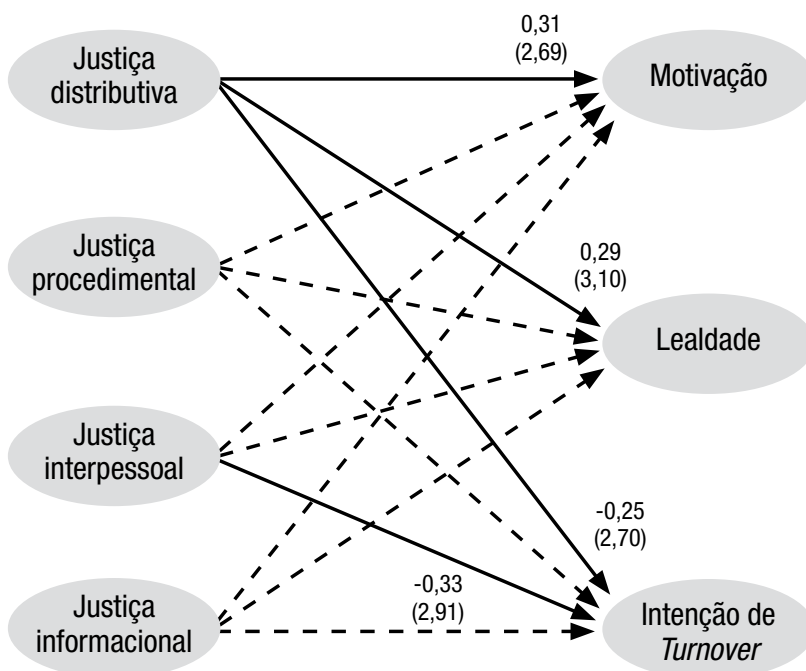

*As linhas tracejadas representam caminhos não significantes ao nível de confiança de $10 \%$ de seus esforços do que por outros fatores. Outro fato que pode explicar esse fenômeno é a característica da amostra do estudo. Como mencionado anteriormente, mais de $80 \%$ da amostra são oriundos do mesmo ramo comercial, cujas especificidades podem ter ocasionado o comportamento analisado acima.

\section{CONSIDERAĢÕES FINAIS}

O principal propósito do trabalho foi entender a relação entre a avaliação de justiça em sua constituição tetradimensional (informacional, procedimental, distributiva e interpessoal) e a motivação, a lealdade e a intenção de turnover de equipes de vendas. Outro objetivo, não menos importante, foi testar um modelo teórico que pudesse servir de referência para futuros estudos. A pesquisa foi realizada por meio de um survey eletrônico com uma amostra final de 194 respondentes, todos profissionais de vendas.

Uma contribuição acadêmica do estudo foi a proposição de uma nova escala para mensuração da 
justiça percebida. A composição fatorial dos itens e as validações convergente, discriminante e da confiabilidade interna indicam que ela é adequada para futuras aplicações no Brasil. A contribuição gerencial, tão importante quanto a acadêmica, aprofunda a compreensão da multidimensionalidade da percepção de justiça, lealdade, motivação e da intenção de turnover. A pesquisa empírica que trata da justiça em vendas é limitada, e evidenciar a influência da justiça percebida na ligação psicológica da equipe de vendas com a empresa (PODASKOFF e MACKENZIE, 1994) pode ser de grande auxílio para a gestão de vendas.

Quanto à contribuição teórica no que se refere à justiça distributiva e interpessoal, pode-se constatar que a forte correlação com a estabilidade da equipe de vendas vai ao encontro a alguns estudos analisados. Concernente à justiça distributiva, remete-se a Deutsch (1975) quanto à percepção de que o recebido, na percepção do destinatário, era algo realmente necessário, assim como a Tyler (1994), no que se refere à equidade. No caso da justiça interpessoal, o clima organizacional (BLODGETT, HILL, TAX, 1997; GOODWIN e ROSS, 1992) revelou-se importante para evitar o turnover por meio do compromisso e compartilhamento das informações.

Quanto à justiça informacional (CROPANZANO e GREENBERG, 1997; GREENBERG, 1993) e processual (TAX, BROWN, CHANDRASSHEKARAN, 1998), a teoria não se confirmou, já que não se encontrou relação com as variáveis consequentes do modelo testado. As características da amostra, com mais de $80 \%$ dos elementos do mesmo ramo, não permitiram avançar nessa parte do estudo. Em decorrência da origem comum dos respondentes, os resultados podem ter refletido uma especificidade do segmento de negócios do qual a amostra faz parte.

A maior limitação metodológica encontrada foi o perfil não probabilístico da amostra aliado à forte endogenia dos respondentes. Essa combinação pode ter influenciado os resultados, no tocante ao não relacionamento entre a justiça informacional e procedimental com as variáveis de resultado. Uma maior heterogeneidade amostral será importante em futuros estudos.

A coleta de dados em corte transversal das variáveis dependentes e independentes pode resultar variância do método (PODSAKOFF e ORGAN, 1986). Os respondentes, percebendo que estão avaliando a relação entre eles e a chefia como justa, poderiam influenciar-se e superestimar as avaliações da lealdade e da motivação e minimizar a avaliação da intenção de trocar de emprego. Para essa limitação, recomenda-se que as coletas dos dados ocorram em momentos distintos.

O estudo não pretendeu encerrar a discussão sobre a influência da justiça percebida sobre a lealdade, motivação e intenção de turnover, mas, sim, deixar em evidência esse importante tema da gestão empresarial.

\section{REFERÊNCIAS}

ADAMS, J. S. Inequity in social exchange. In L. Berkowitz (Ed.), Advances in experimental social psychology, vol. 2. New York: Academic Press, 1965, pp. 267-299

ANDERSON, J. C; GERBING, D. W. Structural equation modeling in practice: a review and recommended two-step approach. Psychological Bulletin, v. 103, n. 5, p. 204-215, 1988.

ANDERSON, E; WEITZ, B. The use of pledges to build and sustain commitment in distribution channels. Journal of Marketing Research, v. 29, n. 1, p. 18-34, 1992.

AQUINO, K. e outros. Integrating justice constructs into turnover process: a test of a referent cognitions model. Academy of Management Journal, v. 40, n. 5, p. 12081227, 1997.

BIES, R. J; MOAG, J. F. Interactional justice: communication criteria of fairness. In: LEWICKI, R. J; SHEPPARD, B. H; BAZERMAN, M. H. (Eds). Research on negotiation in organizations. Greenwich: JAI Press, 1986. p. 43-55.

BIES, R. J; SHAPIRO, D. Interactional fairness judgments: the influence of causal accounts. Social Justice Research, v. 1, n. 2, p. 199-218, 1987.

BLAU, P. Exchange and power in social life. New York: Wiley, 1964.

BlOdGETT, J. G; GRANBOIS, D. H; WALTERS, R. G. The effects of perceived justice on complainants' negative word-of-mouth behavior and repatronage intentions. Journal of Retailing, v. 69, n. 4, p. 399-428, 1993.

BLODGETT, J. G; HILL, D. J; TAX, S. S. The effects of distributive, procedural, and interactional justice on postcomplaint behavior. Journal of Retailing, v. 73, n. 2, p. 185-210, 1997. 
BLODGETT, J. G; WAKEFIELD, K. L; BARNES, J. H. The effects of customer service on consumer complaining behavior. The Journal of Services Marketing, v. 9, n. 4, p. 31-42, 1995.

BRASHEAR, T. G. e outros. An empirical test of trust-bulding process and outcomes in sales manager-salesperson relationships. Journal of the Academy of Marketing Science, v. 31, n. 2, p. 189-200, 2003.

BRASHEAR, T. G; BROOKS, C. M; BOLES, J. S. Distributive and procedural justice in a sales force context: scale development and validation. Journal of Business Research, v. 57, n. 1, p. 86-93, 2004.

BRASHEAR, T. G; MANOLIS, C; BROOKS, C. M. The effects of individual salesperson control on trust and justice. Journal of Business Research, v. 58, n. 3, p. 241-49, 2005.

BROWNE, M. W; CUDECK, R. Alternative ways of assessing model fit. In: BOLLEN, K. A; LONG, L. S. (Orgs) Testing structural equation model. Newbury Park: Sage, 1993. p. 136-162.

CASTILHO, A. Construindo equipes para alto desempenho: fundamentos e técnicas. Rio de Janeiro: Qualitymark, 1998.

CHANG, C. C; DUBINSKI, A. J. Organizational justice in the sales force: a literature review with propositions. Journal of Business-to-Business Marketing, v. 12, n. 1, p. 35-71, 2005.

COLQUITT, J. A. e outros. Justice at the millennium: a meta-analytic review of 25 years of organizational justice research. Journal of Applied Psychology, v. 86, n. 3, p. 425-445, 2001.

CRONIN, J. J; TAYLOR, S. Measuring service quality: a reexamination and extension. Journal of Marketing, v. 56, n. 3, p. 60-63, 1992.

CROPANZANO, R; GREENBERG, J. Progress in organizational justice: tunneling through the maze. In: COOPER, C. L; ROBERTSON, I. T. (Eds) International review of industrial and organizational psychology. New York: John Wiley \& Sons, 1997. p. 317-372.

DECARLO, T. E; LEIGH, T. W. Impact of salesperson attraction on sales managers' attributions and feedback. Journal of Marketing, v. 60, n. 2, p. 47-66, 1996.

DEUTSCH, M. Equity, equality and need: what determines which value will be used as the basis of distributive justice? Journal of Social Issues, v. 31, n. 3, p. 137-50, 1975.
FOLGER, R; GREENBERG, J. Procedural justice: an interpretative analysis of personnel systems. In: ROWLAND, K; FERIS, G. (Eds) Research in personnel and human resources management. Greenwich: JAI Press, 1985. p. 141-183.

FOLGER, R; KONOVSKY, M. A. Effects of procedural and distributive justice on reactions to pay raise decisions. Academy of Management Journal, v. 32, n. 1, p. 115-130, 1989.

GILLILAND, S. W. The perceived fairness of selection systems: an organizational perspective. Academy of Management Review, v. 18, n. 4, p. 694-734, 1993.

GOODHUE, D; LEWIS, W; THOMPSON, R. PLS, small sample size, and statistical power in MIS Research. In: HAWAII INTERNATIONAL CONFERENCE ON SYSTEM SCIENCES, 39, 2006, Washington, DC. Proceedings. Washington, DC: IEEE Computer Society, 2006. 1 CD-ROM.

GOODWIN, C; ROSS, I. Consumer responses to service failures: influence of procedural and interactional fairness perceptions. Journal of Business Research, v. 25, n. 2, p.149-163, 1992.

GREENBERG, J. Equity and workplace status: a field experiment. Journal of Applied Psychology, v. 73, n. 4, p. 603-613, 1988.

GREENBERG, J. Stress fairness to fare no stress: managing workplace stress by promoting organizational justice. Organizational Dynamics, v. 33, n. 4, p. 352-365, 2004.

GREENBERG, J. The intellectual adolescence of organizational justice: you've come a long way, maybe. Social Justice Research, v. 6, n. 1, p. 135-148, 1993.

HAIR JUNIOR, J. F. e outros. Análise multivariada de dados. Tradução Adonai Schlup Sant'Anna e Anselmo Chaves Neto. 5. ed. Porto Alegre: Bookman, 2005.

JARAMILLO, F; MULKI, J. P. Sales effort: the intertwined roles of the leader, customers, and the salesperson. Journal of Personal Selling \& Management, v. 20, n. 1, p. 37-51, 2008.

JÖRESKOG, K; SÖRBOM, D. Lisrel 8: structural equation modeling with the SIMPLIS command language. Licolnwood: Scientific Software International, 1993.

KASHYAP, V. e outros. Developing sales force relationalism: the role of distributive and procedural justice. Jour- 
nal of Personal Selling \& Sales Management, v. 27, n. 3, p. 235-245, 2007.

KERNAN, M. C; HANGES, P. J. Survivor reactions to reorganization: antecedents and consequences of procedural, interpersonal, and informational justice. Journal of Applied Psychology, v. 87, n. 5, p. 916-928, 2002.

KLINE, R. B. Principles and practice of structural equation modeling. New York: The Guilford Press, 2005.

KONOVSKY, M. A. Understanding procedural justice and its impact on business organizations. Journal of Management, v. 26, n. 3, p. 489-511, 2000.

KUMAR, N; SCHEER, L. K; STEENKAMP, J. E. M. The effect of supplier fairness on vulnerable resellers. Journal of Marketing Research, v. 32, n. 1, p. 54-65, 1995.

LIND, E. A; TYLER, T. R. The social psychology of justice. New York: Plenum, 1988.

LU, Y. Toward the micro- and macro-level consequences of interactional justice in cross-cultural joint ventures. $\mathrm{Hu}$ man Relations, v. 59, n. 8, p. 1019-1047, 2006.

MAERTZ JUNIOR e outros. The effects of perceived organizational support and perceived supervisor support on employee turnover. Journal of Organizational Behavior, v. 28, n. 8, p. 1059-1075, 2007.

MALHOTRA, N. K. Pesquisa de marketing: uma orientação aplicada. São Paulo: Bookman, 2006.

MARTIN, J; HARDER, J. W. Bread and roses: justice and the distribution of financial and socioemotional rewards in organizations. Social Justice Research, v. 7, n. 3, p. 241-264, 1994.

MARTINEZ-TUR, V. e outros. Justice perceptions as predictors of customer satisfaction: the impact of distributive, procedural, and interactional justice. Journal of Applied Social Psychology, v. 36, n. 1, p. 110-119, 2006.

MOHR, A; BITNER, M. Mutual understanding between customers and employees in service encounters. Advances in Consumer Research, v. 18, n. 1, p. 611-17, 1991.

NUNNALLY, J. C. Psychometric theory. 2nd ed. New York: McGraw, 1978.
NETEMEYER, R. G. e outros. An investigation into the antecedents of organizational citizenship behaviors in a personal selling context. Journal of Marketing, v. 61, n. 3, p. 85-98, 1997.

OLIVER, R. Cognitive, affective, and attribute bases of the satisfaction response. Journal of Consumer Research, v. 20, n. 1, p. 418-430, 1993.

PIERCY, N. F. e outros. Driving organizational citizenship behaviors and salesperson in-role behavior performance: the role of management control and perceived organizational support. Journal of the Academy of Marketing Science. v. 34, n. 2, p. 244-262, 2006.

PODSAKOFF, P. M; MACKENZIE, S. B. Organizational citizenship behaviors and sales unit effectiveness. Journal of Marketing Research, v. 31, n. 3, p. 351-363, 1994.

PODSAKOFF, P. M; ORGAN, D. W. Self-reports in organizational research: problems and prospects. Journal of Management, n. 12, n. 4, p. 531-544, 1986.

RAMASWAMI, S. N; SINGH, J. Antecedents and consequences of merit pay fairness for industrial salespeople. Journal of Marketing, v. 67, n. 4, p. 46-66, 2003.

SANTOS, C. P; FERNANDES, D. V. D. H. A recuperação de serviços como ferramenta de relacionamento e seu impacto na confiança e lealdade dos clientes. RAE-Revista de Administração de Empresas, v. 48, n. 1, p. 10-24, 2008.

SOTOMAYOR, A. M. S. B. Avaliação de desempenho e compromisso organizacional: a perspectiva da justiça organizacional. Revista Universo Contábil, v. 3, n. 3, p. 87-100, 2007.

TAX, S. S; BROWN, S. W; CHANDRASSHEKARAN, M. Customer evaluations of service complaint experiences: implications for relationship marketing. Journal of Marketing, v. 62, n. 2, p. 60-76, 1998.

THIBAUT, J; WALKER, L. Procedural justice: a psychological analysis. Hillsdale: Erlbaum, 1975.

TYLER, T. R. Psychological models of the justice motive: antecedents of distributive and procedural justice. Journal of Personality and Social Psychology, v. 67, n. 5, p. 850-863, 1994.

WeITZ, B. A; CASTLEBERRY, S. B; TANNER, J. F. Selling: building partnership. Boston: McGraw Hill, 2004. 